\title{
Two barium stars in the Galactic bulge ${ }^{\star}$ (Research Note)
}

\author{
T. Lebzelter ${ }^{1}$, S. Uttenthaler ${ }^{1}$, O. Straniero ${ }^{2}$, and B. Aringer ${ }^{1}$ \\ 1 University of Vienna, Department of Astrophysics, Türkenschanzstrasse 17, 1180 Vienna, Austria \\ e-mail: [thomas.lebzelter; stefan.uttenthaler] @univie.ac.at \\ 2 INAF, Osservatorio Astronomico di Collurania, 64100 Teramo, Italy \\ e-mail: straniero@oa-teramo.inaf.it
}

Received 4 April 2013 / Accepted 30 April 2013

\section{ABSTRACT}

\begin{abstract}
Context. Barium stars conserve important information on the s-process and the third dredge-up in intermediate mass stars. Their discovery in various environments is therefore of great help in testing nucleosynthesis and mixing models.

Aims. Our aim is to analyse two stars with a very strong barium line detected in a large survey of red giants in the Galactic bulge. Methods. Abundance analysis was done comparing synthetic model spectra based on the COMARCS code with our medium resolution spectra. Abundances of Ba, La, Y, and Fe were determined. Beside the two main targets, the analysis was also applied to two comparison stars.

Results. We confirm that both stars are barium stars. They are the first ones of this kind identified in the Galactic bulge. Their barium excesses are among the largest values found up to now. The elemental abundances are compared with current nucleosynthesis and mixing models. Furthermore, we estimate a frequency of barium stars in the Galactic bulge of about $1 \%$, which is identical to the value for disc stars.
\end{abstract}

Key words. stars: late-type - stars: abundances - nuclear reactions, nucleosynthesis, abundances

\section{Introduction}

Low- and intermediate-mass stars manufacture heavy elements via the s-process during their final evolutionary stage, the socalled thermal pulse asymptotic giant branch (TP-AGB). Strong enrichment of these elements in an object therefore normally indicates that the star is already in this phase. However, there is a group of stars showing enhancement in s-process elements while being far away from the TP-AGB phase. Because of the prominent lines of the s-process element barium in their spectra, a sub-group of these objects is called barium-stars (Bidelman \& Keenan 1951). These stars also show unusually strong bands of $\mathrm{CH}$ and $\mathrm{CN}$. The most likely interpretation of these objects is a mass-transfer scenario in a binary system. The more massive companion has already evolved through the TP-AGB phase and deposited material enriched by nucleosynthesis on the less evolved companion, which now appears as a barium star (Boffin \& Jorissen 1988).

Barium stars are interesting objects since they preserve the abundance signature of more massive AGB stars, which is of particular importance for studying metal-poor objects. Their abundance pattern is widely used in calibrating models for s-process nucleosynthesis on the AGB (Busso et al. 1999, 2001). They also give us insight into the mass transfer process in binaries and envelope mixing.

A few hundred barium stars are known in the Milky Way, most of them belonging to the disc population, but some show characteristics of halo kinematics (Gomez et al. 1997). The

\footnotetext{
* Based on observations at the Very Large Telescope of the European Southern Observatory, Cerro Paranal/Chile, under Programme 083.D-0046(A).
}

detection of these objects in various environments allows them to be used as probes for AGB nucleosynthesis at various metallicities (Pereira et al. 2011). The excess of s-process elements in barium stars is typically found in the range $+0.6<[\mathrm{X} / \mathrm{Fe}]<+1.8$ (Allen \& Barbuy 2006) with an increase in the overabundances of the heavy s-elements with decreasing metallicity (Allen \& Barbuy 2006; Pereira et al. 2011). This can be understood in terms of an increased ratio of seed nuclei to free neutrons at higher metallicity and is in agreement with predictions (Busso et al. 1999).

In this paper we report the detection of two additional barium stars. Remarkably, they are located in the Galactic bulge, where no barium stars have been reported yet (see below for some candidates).

\section{Methods}

\subsection{Observations}

The detection of two barium stars appeared as a by-product of our spectroscopic study on a sample of 400 red giants in the Palomar-Gronigen field \# 3 (PG3) of the Galactic bulge. Results on the lithium content have been published in Lebzelter et al. (2012, hereafter Paper I). The same sample has also been used for a detailed study on kinematics and metallicity distribution of red giants in the bulge, see Uttenthaler et al. (2012, hereafter Paper II). Observation of the sample and data reduction have been described in detail in these two papers. We therefore repeat only the basic numbers here.

The observations were done using the FLAMES-GIRAFFE spectrograph at ESO's VLT. A colour-magnitude diagram 


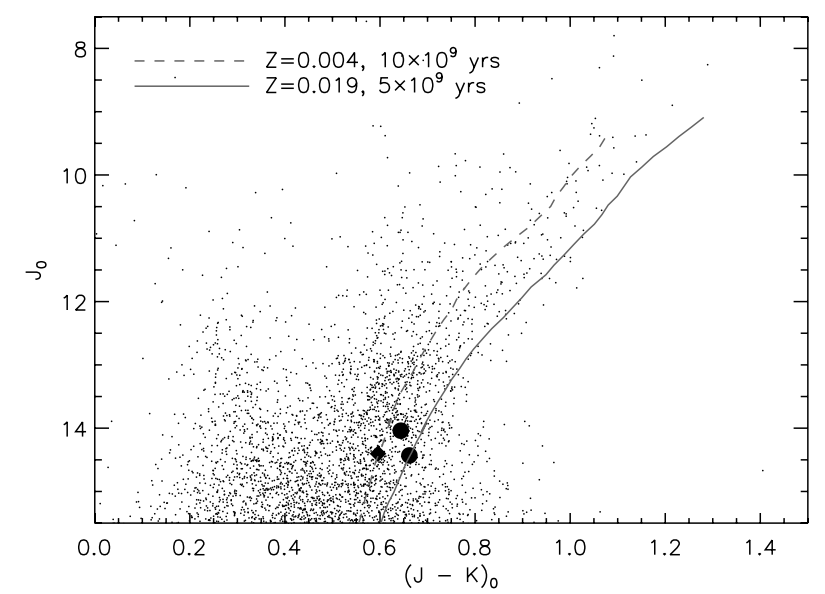

Fig. 1. Dereddened 2MASS colour-magnitude diagram of the barium stars (filled diamond symbols) and the comparison stars (filled circles). The barium star ID 410 basically coincides with its comparison star ID 409 , which is why only the comparison star (the brighter one) is visible here. All other stars shown here are within a 12.5 arcmin diameter field around $(l, b)=\left(0^{\circ},-10^{\circ}\right)$. The solid and dashed grey lines are isochrones from Girardi et al. (2000) with the metallicities and ages as indicated in the legend. They were used to select spectroscopic targets in the bulge (see Paper I) and are truncated at the RGB tip. The RGB bump of these isochrones is at $J_{0} \approx 14$. 0 .

(CMD) of the complete sample has been presented in Fig. 1 of Paper I. The spectra cover the range between 644 and $682 \mathrm{~nm}$ at a resolution of 17000 . The two barium stars reported here were discovered by visual inspection of the spectra, because they showed an outstanding absorption line near $6500 \AA$. Both stars are found at the bottom of the red giant branch. Basic stellar parameters taken from Paper II are summarized in Table 1. The absolute bolometric magnitude was derived from the $K$ magnitude with a bolometric correction term taken from Paper I for a bulge distance modulus of 14.5. We get absolute bolometric luminosities $M_{\text {bol }}$ between 1 m. 2 and 1.6 which is about 5 mag less than the third dredge-up limit (cf. Girardi et al. 2000; Uttenthaler et al. 2007). Our stars are found at a brightness close to the less luminous red clump in the bulge (cf. Uttenthaler et al. 2012). We note, however, that owing to the extension of the bulge and the evolutionary path passing twice through a similar point in the Hertzsprung-Russell-diagram, it is not possible to unambiguously attribute any of our programme stars to that clump. Figure 1 shows the location of the two barium stars in a colour-magnitude diagram of the surrounding bulge field.

Since we have only a single spectrum for each star from our programme, we cannot investigate radial velocity changes as an indication for a possible binary nature. Our field was also observed within the ARGOS survey (Freeman et al. 2013), but unfortunately the barium star candidates were not observed in that survey (Ness, priv. comm.). For comparison, we analysed two more stars in our sample with similar stellar parameters but with no indication of an increased $\mathrm{Ba}$ abundance. Their location in a colour-magnitude diagram of the bulge field is visible in Fig. 1. One of these comparison stars (star 497) was observed by ARGOS, and parameters very similar to our results were found, including the radial velocity $\left(-30.8 \mathrm{~km} \mathrm{~s}^{-1}\right)$.

\subsection{Abundance determination}

As in the previous papers of this series, we used a comparison of observed and synthetic spectra to determine the abundances of selected elements in our two target stars. To compute the synthetic spectra, model atmospheres calculated with the COMARCS code (Aringer et al. 2009) were used. The effective temperatures were set to $4660 \mathrm{~K}, 4810 \mathrm{~K}, 4660 \mathrm{~K}$, and $4610 \mathrm{~K}$, respectively (see Table 1 ). For all four stars a $\log g$ value of 2.5 was used. The microturbulence parameter was set to $1.5 \mathrm{~km} \mathrm{~s}^{-1}$, which was found to give the best overall fit of the observed spectra. The synthetic spectra were computed with a resolution of $R=200000$ and then rebinned to 17000 . A macroturbulence broadening of $3 \mathrm{~km} \mathrm{~s}^{-1}$ was applied.

The metallicity determined in Paper II (Table 1) was taken as a starting value which was further refined in the present analysis. The goal was to determine abundances for Y, Ba, and La. Because of the limited spectral range covered by our observations and the severe line blending, the determination could be based on only one line of these elements. For Ba this was the line at $6498.7 \AA$, for La the line at $6528.9 \AA$, and for $\mathrm{Y}$ the blend with an iron line at $6615 \AA$. All three lines stem from transitions of single ionized atoms. Both the Ba and the La line show a hyperfine structure. This was included in the analysis using the data given in Rutten (1978) for Ba and in Lawler et al. (2001) for La, respectively. Calculations were done in LTE following the calculations of Short \& Hauschildt (2006) showing a very small NLTE effect on the abundance derived from the $\mathrm{Ba}$ line chosen. The solar abundance pattern was taken from Caffau et al. (2008).

For the two barium stars the abundances of all other s-process elements were increased by $0.5 \mathrm{dex}$ in the spectrum calculations, but because of the lack of unblended lines and the limits set by the $\mathrm{S} / \mathrm{N}$ in these comparably faint stars of our sample no attempt was made to fit further element abundances. Contrary to the work done in Paper II which relied primarily on an automatic fitting approach, here the final decision on the best fit was done by visual comparison of model and observation. The clearly visible $\mathrm{CN}$ features in the two barium stars required a slight increase in the $N$ abundance ${ }^{1}$ which is in agreement with expections for this kind of objects. In the analysis of Papers I and II the abundance of $N$ was not altered within the sample. The increased $N$ abundance in these two objects was then obviously compensated by a higher $\mathrm{Fe}$ abundance in the automatic analysis. Therefore, we had to make a refinement of the Fe abundance for these two objects in our present study. However, it seems that this problem affected the metallicity determination in only these two stars, since our analysis of the comparison stars gave the same Fe abundance as the automatic analysis in Paper II.

To estimate the uncertainties of the derived abundances we calculated additional synthetic spectra varying $\log g$ and $T_{\text {eff }}$ by \pm 0.5 and by $\pm 100 \mathrm{~K}$, respectively. Both ranges are the maximum uncertainties in these parameters expected from the analysis in Paper II, and the resulting errors are certainly on the side of caution. In addition, the uncertainty in the exact location of the pseudo-continuum level has been taken into account. The three errors were then combined quadratically. The resulting uncertainties of $[\mathrm{X} / \mathrm{Fe}]$ given in Table 2 also include the uncertainty in metallicity. Note that the abundances are based on the fit of a single line only. The comparably large error in the Y abundance is a result of the studied line being very sensitive to changes in $\log g$. Uncertainties in the $\mathrm{N}$ abundance, as well as uncertainties in the solar reference values, were not considered because of their small effect on the result. We also did a test using a higher

\footnotetext{
1 In principle, the strength of the $\mathrm{CN}$ bands can also be altered by an increase in the $\mathrm{C}$ abundance or by a moderate increase in both abundances. While the best fit was achieved by changing the $N$ abundance, the other two options cannot be ruled out completely.
} 
Table 1. Basic parameters of the analysed targets.

\begin{tabular}{cccccccccccc}
\hline \hline ID & RA (2000) & Dec (2000) & $\begin{array}{c}K \\
{[\mathrm{mag}]}\end{array}$ & $\begin{array}{c}J-K \\
{[\mathrm{mag}]}\end{array}$ & $\begin{array}{c}T_{\text {eff }}[\mathrm{K}] \\
{\left[\begin{array}{llll}\mathrm{RV}_{\text {helio }} \\
{\left[\mathrm{km} \mathrm{s}^{-1}\right]}\end{array}\right.}\end{array}$ & {$[\mathrm{Fe} / \mathrm{H}]$} & {$[\alpha / \mathrm{Fe}]$} & B.C. & $M_{\text {bol }}$ & Comment \\
\hline 410 & $18: 27: 00.8$ & $-33: 44: 18$ & 13.448 & 0.725 & 4659 & +24.1 & -0.28 & +0.23 & 2.2 & 1.2 & Barium star \\
490 & $18: 26: 50.9$ & $-33: 47: 39$ & 13.856 & 0.675 & 4810 & -2.5 & -0.56 & +0.23 & 2.2 & 1.5 & Barium star \\
409 & $18: 26: 18.9$ & $-33: 42: 21$ & 13.446 & 0.725 & 4665 & +0.5 & -0.04 & +0.14 & 2.2 & 1.2 & Comparison star \\
497 & $18: 27: 19.4$ & $-33: 53: 46$ & 13.816 & 0.739 & 4612 & -30.9 & -0.52 & +0.20 & 2.3 & 1.6 & Comparison star \\
\hline
\end{tabular}

Notes. All data are taken from Paper II. $M_{\text {bol }}$ calculated using $(m-M)=14.5$ and a bolometric correction (B.C.) as in Paper I.

Table 2. Derived abundances for selected elements.

\begin{tabular}{lcccccccc}
\hline \hline $\mathrm{ID}$ & {$[\mathrm{Fe} / \mathrm{H}]$} & $\Delta[\mathrm{Fe} / \mathrm{H}]$ & {$[\mathrm{Ba} / \mathrm{Fe}]$} & $\Delta[\mathrm{Ba} / \mathrm{Fe}]$ & {$[\mathrm{La} / \mathrm{Fe}]$} & $\Delta[\mathrm{La} / \mathrm{Fe}]$ & {$[\mathrm{Y} / \mathrm{Fe}]$} & $\Delta[\mathrm{Y} / \mathrm{Fe}]$ \\
\hline 410 & -0.5 & 0.2 & +2.3 & 0.4 & +1.8 & 0.5 & 0.9 & 0.5 \\
490 & -0.7 & 0.1 & +1.8 & 0.2 & +1.5 & 0.3 & 0.8 & 0.5 \\
409 & -0.1 & 0.2 & -0.1 & 0.4 & 0.0 & 0.4 & 0.0 & 0.6 \\
497 & -0.5 & 0.2 & -0.2 & 0.4 & 0.0 & 0.4 & 0.0 & 0.6 \\
\hline
\end{tabular}
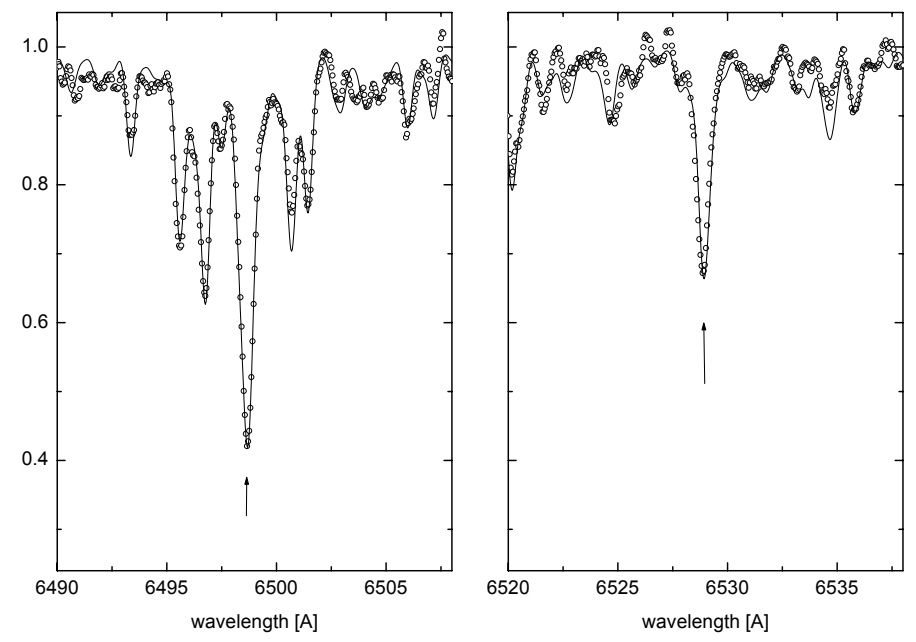

Fig. 2. Ba II line (left) and La II line (right, marked by an arrow) used to derive the corresponding element abundances. The observed spectrum of star 490 is shown by open symbols; the solid line gives the best fit model.

$\mathrm{C} / \mathrm{O}$ ratio of 0.9 in the model, which may be more appropriate for a highly evolved AGB star transfering mass on the companion. This change is hardly visible in the model spectrum in the selected wavelength region, and thus has no effect on the derived s-process element abundances.

\section{Results}

Figure 2 shows an example of the model fit of the observed spectra. The overall agreement is satisfying. The results are summarized in Table 2. The elments $\mathrm{Ba}$ and $\mathrm{La}$ are strongly enhanced in both barium stars. A less expressed enhancement is also seen in $\mathrm{Y}$ with a larger error bar. As mentioned above the iron abundance is slightly different from the values derived in Paper II. We also note that the fit of the Sc II line at $6606.5 \AA$ can be improved when reducing the abundance of this element by about 0.4 dex in these objects. From Paper I we know that the stars do not show any significant abundance of $\mathrm{Li}$.

The analysis also confirmed that the two comparison stars were not barium stars. None of the tested elements shows any considerable enhancement above the scaled solar value in these cases.
In both stars 410 and 490, barium is more enhanced than lanthanum, although the difference is within the error bars. A key indicator used for the comparison of TP-AGB models with observations is the ratio between the light and the heavy s-process elements. Classicaly, this ratio is formed using the $\mathrm{Y}$ and $\mathrm{Zr}$ abundance on the one hand and the $\mathrm{La}, \mathrm{Nd}$, and $\mathrm{Sm}$ abundance on the other (e.g. Husti et al. 2009). We can only give a simplified version here forming the ratio between $[\mathrm{Y} / \mathrm{Fe}]$ and $[\mathrm{La} / \mathrm{Fe}]$. This gives for $[\mathrm{hs} / \mathrm{ls}]$ values of 0.9 and 0.7 for star 410 and star 490, respectively. The considerable uncertainty in both abundances limits the reliability of this result. However, errors in temperature and $\log g$ alter the abundances of La and $\mathrm{Y}$ in the same direction, thus giving a combined uncertainty of only about 0.3 dex.

\section{Discussion}

To our knowledge, this is one of the first detections of Ba stars in the Galactic bulge. Johnson et al. (2012) detected one giant in their sample of 69 bulge stars with a remarkable overabundance of $[\mathrm{La} / \mathrm{Fe}]=+1.43 \pm 0.32$, and $[\mathrm{Fe} / \mathrm{H}]=-0.56$. That star is also enriched in $\mathrm{Zr}$ and Nd. However, Johnson et al. (2012) did not discuss this object further. Using the star's $K$ magnitude and the same approach as for our targets, we get an $M_{\mathrm{bol}}=-0.9$. This star (2MASS 18174742-3348098) is, thus, much brighter than our targets but still less luminous than the 3DUP limit. We therefore consider this object a barium star as well. Cavallo et al. (2003) found enhancements of several s-process elements in a few bulge stars studied using a microlensing technique. Among them is an interesting dwarf star, 99-BLG-1, which shows a strong enhancement in $\mathrm{Ru}, \mathrm{La}$, and $\mathrm{Ce}$, but surprisingly not in $\mathrm{Ba}$, which is solar in this object. We note, however, that Cavallo et al. (2003) did not apply a correction for hfs splitting of the analysed lines, therefore the actual error bar may be larger than their given value of 0.2 dex.

With $[\mathrm{Fe} / \mathrm{H}]$ of about -0.5 , our two barium stars are located close to the metallicity where the maximum enhancement for the second s-process peak (Ba peak) is expected (cf. Fig.12 in Busso et al. 1999). The measured overabundances for $\mathrm{Ba}$ and $\mathrm{La}$ agree well with the predictions for a $1.5 M_{\odot}$ TP-AGB star of that metallicity with the standard choice of the ${ }^{13} \mathrm{C}$ pocket efficiency (Husti et al. 2009). On the contrary, the measured abundances for $\mathrm{Y}$ are a bit lower than the model calculation. More recent calculations by Cristallo et al. (2011) include a simultaneous solution 
of the AGB evolution of the physical structure and the chemical evolution ${ }^{2}$. For the metallicity given in Table 2 we find a good agreement of our $[\mathrm{Ba} / \mathrm{Fe}]$ and $[\mathrm{La} / \mathrm{Fe}]$ values for an AGB star around 2.0 to $2.5 M_{\odot}$, which would be the companion of the barium star. The Y abundance predicted by these models agrees with our measurements within the error bars.

Compared to the measured excesses in literature studies on larger samples (Allen \& Barbuy 2006; Smiljanic et al. 2007; Pereira et al. 2011), our stars are found in the top range for the $\mathrm{Ba}$ and $\mathrm{La}$ abundance excesses for all barium stars. In particular, star 410 may be the star with the highest Ba overabundance detected up to now. Compared to the Y abundance excesses found for barium stars of that metallicity by Allen \& Barbuy (2006), the two objects studied here are found at the lower end of the distribution.

The measured abundance of $\mathrm{Y}, \mathrm{Ba}$, and $\mathrm{La}$ in a barium star depends also on the dilution factor describing the change of surface abundances due to mixing of the accreted material with the deeper layers of the original stellar atmosphere. The large excess of $\mathrm{Ba}$ and La observed seems to be compatible with the hypothesis that no major mixing event has occurred in the stars since the accretion took place. The derived $\log g$ value suggests that both our barium stars are indeed giants. We may speculate that the mass transfer from the TP-AGB star happened after the major mixing event of the first dredge-up. According to Girardi et al. (2000), the total life times of a star of 2 and $2.5 M_{\odot}$ are $1.1 \times 10^{9}$ and $5.9 \times 10^{8}$ years, respectively. If this hypothesis is correct and the accretion process occurred after the first dredge-up of the companion, the mass of the barium star has to be chosen such that it reaches the RGB within the total life time of the donor star. This would mean that our two barium stars, in particular star 410 , have a mass larger than $1.7 M_{\odot}$. Such a mass estimate would nicely agree with the existence of an intermediate population in the bulge suggested, e.g. by Uttenthaler et al. (2007) to explain the existence of the third dredge-up indicator Tc in bulge AGB stars. However, this scenario is affected by the various uncertainties in the data, so that a final conclusion cannot be drawn.

An alternative conclusion would be that the calculated AGB yields are underestimated. In that case, it would imply that the third dredge-up is deeper than estimated in the calculations of, e.g. Cristallo et al. (2011), and/or the mass loss rate is lower than assumed. Concerning the mass loss rate, Cristallo et al. (2009) expect an increase in the La yields of $24 \%$ if the mass loss rate is reduced by a factor of 2 . A more efficient third dredge-up, resulting from a larger mixing length parameter in the models, also leads to a significant increase of the predicted La abundance. The fact that both of our barium stars come with a high Ba abundance may suggest that the second scenario, an underestimate of AGB yields by the models, is more probable since the probability that both stars are found immediately after the mass transfer from the companion is expected to be rather low. However, it is remarkable that there are not more stars known with a barium abundance similar to star 410 .

Because of the surprisingly low abundance of $\mathrm{Y}$, the ratio [hs/ls] is clearly higher in our two barium stars than the value expected from the models (cf. Fig. 7 in Busso et al. 2001). However, it is not completely out of the range of observed values, and considering the error bar it may well fit within the range of the models. An extended study based on more lines and including an abundance analysis of further elements from the $\mathrm{Y} / \mathrm{Zr}$-peak would be needed to give a proper [hs/ls] ratio. Its obvious advantage for a comparison with stellar nucleosynthesis models would be its independency of the uncertainties of dredge-up efficiency, mass loss rate, and dilution factor.

The two Ba stars detected here and the one from Johnson et al. (2012) are probably members of the metal-poor population identified previously in the bulge (Paper II, Hill et al. 2011). In Paper II we found that the transition between the metal-poor and metal-rich population is at $[\mathrm{Fe} / \mathrm{H}] \sim-0.20$, meaning that 243 giants from that sample belong to the metal-poor population. In Johnson's paper, 31 bulge RGB stars are more metalpoor than this limit, one of which is the Ba star discussed above. This means that in the metal-poor population three out of 274 giants are Ba stars, giving a Ba-star fraction of $\sim 1 \%$. This is identical with the findings from the disc field (Izzard et al. 2010; MacConnell et al. 1972). Ba stars in the metal-rich bulge population yet remain to be identified. It is thus possible that the fraction of binaries that allow for the formation of Ba stars in that population is lower than in the metal-poor population and the disc. This would be in agreement with expectations since metal poor stars are known to show a more efficient dredge-up during the AGB phase. However, at the moment there are only very few studies of the s-element content of bulge stars available.

Acknowledgements. The work of T.L. has been supported by the Austrian Science Fund under project number P23737-N16. S.U. acknowledges support from the Austrian Science Fund (FWF) under project P 22911-N16. The authors thank Martin Stift for helpful discussions on the hyperfine structure of lines, and Melissa Ness for providing the ARGOS results for one of the comparison stars.

\section{References}

Allen, D. M., \& Barbuy, B. 2006, A\&A, 454, 895

Aringer, B., Girardi, L., Nowotny, W., Marigo, P., \& Lederer, M. T. 2009, A\&A, 503,913

Bidelman, W. P., \& Keenan, P. C. 1951, ApJ, 114, 473

Boffin, H. M. J., \& Jorissen, A. 1988, A\&A, 205, 155

Busso, M., Gallino, R., \& Wasserburg, G. J. 1999, ARA\&A, 37, 239

Busso, M., Gallino, R., Lambert, D. L., Travaglio, C., \& Smith, V. V. 2001, ApJ, 557,802

Caffau, E., Ludwig, H.-G., Steffen, M., et al. 2008, A\&A, 488, 1031

Cavallo, R. M., Cook, K. H., Minniti, D., \& Vandehei, T. 2003, in SPIE Conf. Ser. 4834, ed. P. Guhathakurta, 66

Cristallo, S., Straniero, O., Gallino, R., et al. 2009, ApJ, 696, 797

Cristallo, S., Piersanti, L., Straniero, O., et al. 2011, ApJS, 197, 17

Freeman, K., Ness, M., Wylie-de-Boer, E., et al. 2013, MNRAS, 428, 3660

Girardi, L., Bressan, A., Bertelli, G., \& Chiosi, C. 2000, A\&AS, 141, 371

Gomez, A. E., Luri, X., Grenier, S., et al. 1997, A\&A, 319, 881

Hill, V., Lecureur, A., Gómez, A., et al. 2011, A\&A, 534, A80

Husti, L., Gallino, R., Bisterzo, S., Straniero, O., \& Cristallo, S. 2009, PASA, 26,176

Izzard, R. G., Dermine, T., \& Church, R. P. 2010, A\&A, 523, A10

Johnson, C. I., Rich, R. M., Kobayashi, C., \& Fulbright, J. P. 2012, ApJ, 749, 175

Lawler, J. E., Bonvallet, G., \& Sneden, C. 2001, ApJ, 556, 452

Lebzelter, T., Uttenthaler, S., Busso, M., Schultheis, M., \& Aringer, B. 2012, A\&A, 538, A36 (Paper I)

MacConnell, D. J., Frye, R. L., \& Upgren, A. R. 1972, AJ, 77, 384

Pereira, C. B., Sales Silva, J. V., Chavero, C., Roig, F., \& Jilinski, E. 2011, A\&A, 533, A51

Rutten, R. J. 1978, Sol. Phys., 56, 237

Short, C. I., \& Hauschildt, P. H. 2006, ApJ, 641, 494

Smiljanic, R., Porto de Mello, G. F., \& da Silva, L. 2007, A\&A, 468, 679

Uttenthaler, S., Hron, J., Lebzelter, T., et al. 2007, A\&A, 463, 251

Uttenthaler, S., Schultheis, M., Nataf, D. M., et al. 2012, A\&A, 546, A57 (Paper II)

\footnotetext{
2 http://fruity.oa-teramo.inaf.it/
} 